\title{
Inventory control model based on multi-attribute material classification: An integrated grey-rough set and probabilistic neural network approach
}

\author{
Zhang, Z.L. ${ }^{\mathrm{a}, \mathrm{b},{ }^{*}}$, Wang, Y.F. ${ }^{\mathrm{C}}$, Li, Y. ${ }^{\mathrm{d},{ }^{*}}$ \\ ${ }^{a}$ Northeast Forestry University, College of Economics and Management, Harbin, P.R. China \\ ${ }^{\mathrm{b}}$ Jiamusi University, College of Economics and Management, Jiamusi, P.R. China \\ ‘Jiamusi University, School of Clinical Medicine, Jiamusi, P.R. China \\ dLiaoning University, Business School, Shenyang, P.R. China
}

\section{A B S T R A C T}

Efficient and reasonable inventory control can help enterprises improve inventory management efficiency, reduce inventory cost, and ensure the full utilization of resources. Considering that there are many attributes of material, different materials have different effects on enterprises. A multi-attribute material classification model based on grey rough set and probabilistic neural network is proposed, and an inventory control strategy model based on material classification is constructed according to the characteristics of different types of material. Based on the construction of the relevant models, taking the inventory materials of sample Enterprise $A$ as an example, the grey rough set algorithm is used to reduce the redundant material attributes, and the sample data of normalized reduction attributes are used to classify and discriminate the materials by probabilistic neural network. The results are simulated by MATLAB to obtain the efficient and reasonable classification of the materials of enterprises. Finally, with the sample data of different types of representative materials, a matching model of inventory control strategy based on material classification is applied in practice, and the applicability and feasibility of the model are illustrated, providing a scientific basis for enterprises to make decisions on material management and inventory control.
\end{abstract}

\section{ARTICLE INFO}

\section{Keywords:}

Inventory control strategy;

Modelling;

Material classification;

Grey rough set;

Probabilistic neural network

*Corresponding author:

jeanneettee@163.com

(Zhang, Z.L.)

lyfly68@163.com

(Li, Y.)

Article history:

Received 30 November 2018

Revised 4 February 2019

Accepted 24 February 2019

\section{References}

[1] Gholamian, M.R., Heydari, M. (2017). An inventory model with METRIC approach in location-routing-inventory problem, Advances in Production Engineering \& Management, Vol. 12, No. 2, 115-126, doi: 10.14743/apem2017. $\underline{2.244}$.

[2] Jin, X.-W. (1995). An inventory control model for multistage demand imbalance: comment on SM-I method, Journal of Quantitative \& Technical Economics, No. 9, 48-51, doi: 10.13653/i.cnki.jate.1995.09.010.

[3] Tan, Z.D. (2014). Efficient warehouse control supply and logistics "regulating valve", Petroleum \& Petrochemical Material Procurement, Vol. 4, No. 3, 28-29.

[4] Katsios, D., Xanthopoulos, A.S., Koulouriotis, D.E., Kiatipis, A. (2018). A simulation optimisation tool and its production/inventory control application, International Journal of Simulation Modeling, Vol. 17, No. 2, 257-270, doi: 10.2507/IJSIMM17(2)425.

[5] Xiao, Y.-Y., Zhang, R.-Q., Kaku I. (2011). A new approach of inventory classification based on loss profit, Expert Systems with Applications, Vol. 38, No. 8, 9382-9391, doi: 10.1016/j.eswa.2011.01.127.

[6] Kabir, G., Hasin, M.A.A. (2013). Multi-criteria inventory classification through integration of fuzzy analytic hierarchy process and artificial neural network, International Journal of Industrial and Systems Engineering, Vol. 14, No. 1, 74-103, doi: 10.1504/IJISE.2013.052922. 
[7] Šarić, T., Šimunović, K., Pezer, D., Šimunović, G., (2014). Inventory classification using multi-criteria ABC analysis, neural networks and cluster analysis, Tehnički Vjesnik - Technical Gazette, Vol. 21, No. 5, 1109-1115.

[8] Douissa, M.R., Jabeur, K. (2016). A new model for multi-criteria ABC inventory classification: PROAFTN method, Procedia Computer Science, No. 96, 550-559, doi: 10.1016/i.procs.2016.08.233.

[9] May, B.I., Atkinson, M.P., Ferrer, G. (2017). Applying inventory classification to a large inventory management system, Journal of Operations and Supply Chain Management, Vol. 10, No. 1, 68-86, doi: 10.12660/joscmv10n1 p68-86.

[10] $\mathrm{Hu}$, J.-F., He, L.-L., Zhou, Q.-Y. (2015). Research on ABC inventory classification based on cluster analysis, Industrial Control Computer, Vol. 28, No. 3, 147-148, doi: 10.3969/j.issn.1001-182X.2015.03.062.

[11] Chen, Z., Tang, S.-H., Shan, M.-Y. (2008). Study on the strategy of inventory classified management matrix in manufacturing enterprise, The Theory and Practice of Finance and Economics. Vol. 29, No. 6, 105-109, doi: 10.3969/j.issn.1003-7217.2008.06.023.

[12] Strijbosch, L.W.G., Moors, J.J.A. (2006). Modified normal demand distributions in $(R, S)$-inventory control, European Journal of Operational Research, Vol. 172, No. 1, 201-212, doi: 10.1016/j.ejor.2004.10.002.

[13] Rossetti, M.D., Shbool, M., Varghese, V., Pohl, E. (2013). Investigating the effect of demand aggregation on the performance of an (R, Q) inventory control policy, In: Proceedings of the 2013 Winter Simulation Conference: Simulation: Making Decisions in a Complex World, Washington, USA, 3318-3329, doi: 10.1109/WSC.2013. 6721 $\underline{696}$.

[14] Liu, A.-Y. (2015). Research on inventory control of service spare parts of electronic manufacturing industry company, Master's thesis, Taiyuan University of Technology, School of economics and management, Taiyuan. China.

[15] Liu, Z.-Y., Jiang, S.-M. (2017). The ordering strategy research of core material of W auto parts manufacturing enterprise, Logistics Engineering and Management, Vol. 39, No. 11, 130-132.

[16] Zhang, J. (2007). Research on inventory control strategy under the condition of random requirement, Pioneering with Science \& Technology Monthly, No. 11, 78-79, doi: 10.3969/j.issn.1672-2272.2007.11.039.

[17] Güler, M.G., Bilgiç, T., Güllü, R. (2015). Joint pricing and inventory control for additive demand models with reference effects, Annals of Operations Research, Vol. 226, No. 1, 255-276, doi: 10.1007/s10479-014-1706-3.

[18] Zhao, C. (2016). Optimization and simulation of multi-echelon inventory in supply chain under stochastic demand based on control theory, Doctoral thesis, University of International Business and Economics, School of International Trade and Economics, Beijing, P.R. China.

[19] Gocken, M., Dosdogru, A.T., Boru, A. (2017). Optimization via simulation for inventory control policies and supplier selection, International Journal of Simulation Modeling, Vol. 16, No. 2, 241-252, doi: 10.2507/IJSIMM16(2) $\underline{5.375}$.

[20] Strijbosch, L.W.G., Syntetos, A.A., Boylan, J.E., Janssen, E. (2011). On the interaction between forecasting and stock control: The case of non-stationary demand, International Journal of Production Economics, Vol. 133, No. 1, 470-480, doi: 10.1016/j.ijpe.2009.10.032.

[21] Rahdar, M., Wang, L., Hu, G. (2018). A tri-level optimization model for inventory control with uncertain demand and lead time, International Journal of Production Economics, Vol. 195, 96-105, doi: 10.1016/i.ijpe.2017.10.011.

[22] Li, B., Wang, H., Yang, J., Guo, M. (2011). Belief-rule-based inference method for inventory control, Journal of Huazhong University of Science and Technology (Natural Science Edition), Vol. 39, No. 7, 76-79, doi: 10.13245/ j.hust.2011.07.001.

[23] Yan, X.-J., Zhang, M.-K., Chen, Z.-Y. (2016). Studies on risk control of materials purchasing using the MSPC method, Journal of Applied Statistics and Management, Vol. 35, No. 2, 360-368, doi: 10.13860/j.cnki.sltj.20160123-001.

[24] Li, F. (2011). Research on material classification of wind-electric assemble plant, Master's thesis, Chongqing University, College of Mechanical Engineering, Chongqing, P.R. China.

[25] Han, S., Lu, J., Wei, C., Liu, Z. (2014). Research of classification method of materials and equipments of coal enterprise based on probabilistic neural network, Industry and Mine Automation, Vol. 40, No. 4, 38-41, doi: 10.13272/ j.issn.1671-251x.2014.04.009.

[26] Lee, H.L., Billington, C. (1993). Material management in decentralized supply chains, Operations Research, Vol. 41, No. 5, 835-847, doi: 10.1287/opre.41.5.835.

[27] Mohanty, R.P., Deshmukh, S.G. (2001). Reengineering of materials management system: A case study, International Journal of Production Economics, Vol. 70, No. 3, 267-278, doi: 10.1016/S0925-5273(00)00071-2.

[28] Hallikas, J., Karvonen, I., Pulkkinen, U., Virolainen, V.-M., Tuominen, M. (2004). Risk management processes in supplier networks, International Journal of Production Economics, Vol. 90, No. 1, 47-58, doi: 10.1016/j.ijpe.2004. 02.007.

[29] Partovi, F.Y., Anandarajan, M. (2002). Classifying inventory using an artificial neural network approach, Computers \& Industrial Engineering, Vol. 41, No. 4, 389-404, doi: 10.1016/S0360-8352(01)00064-X.

[30] Haykin, S. (2008). Neural networks and learning machine, 3rd edition, Pearson Education, Inc., Upper Saddle River, Pearson Prentice Hall, New Jersey. 


\title{
Model nadzora inventarja, ki temelji na klasifikaciji materiala $z$ več atributi: Integriran pristop $z$ uporabo sive grobe množice in verjetnostne nevronske mreže
}

\author{
Zhang, Z.L. ${ }^{\mathrm{a}, \mathrm{b},{ }^{*}}$, Wang, Y.F. ${ }^{\mathrm{C}}$, Li, Y. ${ }^{\mathrm{d},{ }^{*}}$ \\ ${ }^{a}$ Northeast Forestry University, College of Economics and Management, Harbin, P.R. China \\ ${ }^{\mathrm{b}}$ Jiamusi University, College of Economics and Management, Jiamusi, P.R. China \\ `Jiamusi University, School of Clinical Medicine, Jiamusi, P.R. China \\ dLiaoning University, Business School, Shenyang, P.R. China
}

\section{POVZETEK}

Učinkovit in razumen nadzor zalog lahko podjetjem pomaga izboljšati učinkovitost upravljanja zalog, zmanjšati stroške zalog in zagotoviti polno izrabo virov. Glede na to, da obstaja veliko lastnosti materiala, imajo različni materiali različne učinke na podjetja. Predlagan je model klasifikacije materiala z več atributi, ki temelji na sivi grobi množici in verjetnostni nevronski mreži, model strategije nadzora inventarja, ki temelji na klasifikaciji materiala, pa je zasnovan glede na značilnosti različnih vrst materiala. Na podlagi izdelave ustreznih modelov, če vzamemo za primer materiale inventarja vzorčnega podjetja $A$, se algoritem sive grobe množice uporablja za zmanjšanje odvečnih atributov materiala, vzorčni podatki normaliziranih atributov zmanjšanja pa se uporabljajo za klasifikacijo in razlikovanje materiala $\mathrm{z}$ verjetnostno nevronsko mrežo. MATLAB simulira rezultate za učinkovito in razumno klasifikacijo materialov podjetij. Nazadnje se z vzorčnimi podatki različnih vrst reprezentativnih materialov v praksi uporabi ujemajoč model strategije nadzora zalog, ki temelji na klasifikaciji materialov. Ponazorita se uporabnost in izvedljivost modela, ki podjetjem zagotavlja znanstveno podlago za odločanje o upravljanju materiala in nadzoru zalog.
\end{abstract}

\section{PODATKI O ČLANKU}

\section{Ključne besede:}

Strategija nadzora zalog;

Modeliranje;

Klasifikacija materiala;

Siva groba množica;

Verjetnostna nevronska mreža

*Kontaktna oseba:

jeanneettee@163.com

(Zhang, Z.L.)

lyfly68@163.com

(Li, Y.)

Zgodovina članka:

Prejet 30. novembra 2018

Popravljen 4. februarja 2019

Sprejet 24. februarja 2019 